\title{
Quintessence and Spontaneous Leptogenesis
}

\author{
Mingzhe Li, Xiulian Wang, Bo Feng and Xinmin Zhang \\ Institute of High Energy Physics, \\ Chinese Academy of Science, PO Box 918-4, Beijing 100039, \\ P. R. China
}

\begin{abstract}
We propose in this paper a scenario of spontaneous baryogenesis in cosmological models of Quintessence by introducing a derivative coupling of the Quintessence scalar $Q$ to the baryon current $J_{B}^{\mu}$ or the current of the baryon number minus lepton number $J_{B-L}^{\mu}$. We find that with a dimension5 operator $\partial_{\mu} Q J_{B-L}^{\mu}$ suppressed by the Planck mass $M_{p l}$ or the Grand Unification Scale $M_{G U T}$, baryon number asymmetry $n_{B} / s \sim 10^{-10}$ can be naturally explained via leptogenesis. We study also the isocurvature baryon number fluctuation generated in our model.
\end{abstract}

PACS numbers: 98.80.Cq

Evidence is increasing that Universe is spatially flat and accelerating at present time [1]. The simplest account of the cosmic acceleration seems to be a remnant small cosmological constant $\Lambda$, however many physicists are attracted by the idea that a new form of matter is causing the cosmic acceleration. This new form of matter is not clustered gravitationally on the scale of the galaxy clusters and has been usually called dark energy or Quintessence 225.

For Quintessence, typically one assumes the existence of a scalar field $Q$ (or multi scalar fields) which is taken to be homogeneous in space and via its kinetic and potential energy contributions to the energy-momentum tensor tuned to provide an equation of state leading to accelerated expansion, beginning to dominate the matter content of the Universe today. Models of Quintessence can have interesting cosmological properties including tracking behavior.

Generally minimal or non-minimal couplings of Quintessence with gravitation are considered in the literature [6]. Being a dynamical field, Quintessence is expected to have interactions with the ordinary matters [7], however as argued in Refs. [8] for most of cases the couplings are strongly constrained. But there are exceptions. For example, Carroll [8] has considered an interaction of form $Q F_{\mu \nu} \tilde{F}^{\mu \nu}$ with $F_{\mu \nu}$ being the electromagnetic field strength tensor and studied its implication on the rotation of the plane of polarization of light coming from distant sources. In this paper we introduce a type of interaction of Quintessence with the matter, which in terms of an effective lagrangian is given by

$$
\mathcal{L}_{\text {eff }}=\frac{c}{M} \partial_{\mu} Q J^{\mu}
$$

where $M$ is the cut-off scale which for example could be the Planck mass $M_{p l}$ or the scale of Grand Unification Theory $M_{G U T}$, and $c$ is the coupling constant which characterizes the strength of Quintessence interacting with the ordinary matter in the Standard Model of the electroweak theory. Specifically we take in this paper $J^{\mu}$ to be the baryon current $J_{B}^{\mu}$ or the current of baryon number minus lepton number $J_{B-L}^{\mu}$, and study their implications on baryogenesis. The lagrangian in Eq.(1) involves derivative and obeys the symmetry $Q \rightarrow Q+$ constant [8], so the Quintessence potential will not be modified by the quantum corrections.

The mechanism of generating the baryon number asymmetry in this paper follows closely the spontaneous baryogenesis [9, 10]. The term in Eq.(11), when $\dot{Q}$ is non-zero during the evolution of spatial flat Friedmann-Robertson-Walker Universe, violates CPT invariance and generates an effective chemical potential $\mu_{b}$ for baryons, i.e.,

$$
\begin{aligned}
& \frac{c}{M} \partial_{\mu} Q J_{B}^{\mu} \rightarrow c \frac{\dot{Q}}{M} n_{B}=c \frac{\dot{Q}}{M}\left(n_{b}-n_{\bar{b}}\right), \\
& \mu_{b}=c \frac{\dot{Q}}{M}=-\mu_{\bar{b}} .
\end{aligned}
$$

In thermal equilibrium the baryon number asymmetry is given by (when $T \gg m_{b}$ )

$$
n_{B}=\frac{g_{b} T^{3}}{6}\left(\frac{\mu_{b}}{T}+\mathcal{O}\left(\frac{\mu_{b}}{T}\right)^{3}\right) \simeq c \frac{g_{b} \dot{Q} T^{2}}{6 M},
$$

where $g_{b}$ counts the internal degree of freedom of the baryon. Using the familiar expression for entropy density

$$
s=\frac{2 \pi^{2}}{45} g_{\star} T^{3},
$$

we arrive at the final expression for the baryon to entropy ratio 


$$
n_{B} / s \simeq \frac{15 c}{4 \pi^{2}} \frac{g_{b} \dot{Q}}{g_{\star} M T}
$$

$\dot{Q}$ in Eq.(5) can be obtained by solving the equation of motion of Quintessence given below

$$
\ddot{Q}+3 H \dot{Q}+V^{\prime}(Q)=-\frac{c}{M}\left(\dot{n}_{B}+3 H n_{B}\right)
$$

where $\mathrm{H}$ is the Hubble constant and $V(Q)$ is the potential of Quintessence field. For the radiation dominated era the Hubble constant is

$$
H=\frac{1}{2 t}=1.66 g_{\star}^{1 / 2} \frac{T^{2}}{M_{p l}} .
$$

The right-handed side of Eq.(6) is about $-\frac{c g_{b}}{6} \frac{T^{2}}{M^{2}}(\ddot{Q}+H \dot{Q})$ and can be neglected unless in the very early universe when the temperature $T$ is close to the cut-off scale.

In this paper we consider a model which has the tracking property, where the potential has a modified exponential form 11$]$,

$$
V(Q)=f(Q) e^{-\frac{\lambda}{m_{p l}} Q}
$$

In Eq.(8), $f(Q)$ is a function of $\mathrm{Q}$ which is required to change more slowly than $e^{-\frac{\lambda}{m_{p l}} Q}$ in the regime of tracking. For the detailed discussions on the specific form of $f(Q)$ and model properties we refer to Ref. [11]. Here we simply summarize the salient features of this model. For $\lambda^{2}>3\left(1+w_{B}\right)$ with $w_{B}$ being the ratio of the pressure to the energy density of the background fluid, this model has an attractor solution [12 15,

$$
\begin{aligned}
\Omega_{Q} & \equiv \frac{\rho_{Q}}{\rho_{Q}+\rho_{B}}=\frac{3}{\lambda^{2}}\left(1+w_{B}\right), \\
w_{Q} & =w_{B} .
\end{aligned}
$$

During radiation dominated era, $\rho_{B}=\frac{\pi^{2}}{30} g_{\star} T^{4}$ and $w_{B}=1 / 3$, so we have

$$
\begin{aligned}
\rho_{Q} & \simeq \frac{2 \pi^{2}}{15\left(\lambda^{2}-4\right)} g_{\star} T^{4}, \\
\dot{Q} & \simeq \frac{4 \pi}{3 \sqrt{10\left(\lambda^{2}-4\right)}} g_{\star}^{1 / 2} T^{2} .
\end{aligned}
$$

Thus we obtain the ratio of baryon number to entropy, which is given by

$$
\frac{n_{B}}{s} \simeq \frac{5 c}{\pi \sqrt{10\left(\lambda^{2}-4\right)}} g_{b} g_{\star}^{-1 / 2} \frac{T}{M} .
$$

Taking $\lambda^{2}>20$ 14,15 constrained by nucleosynthesis, and $g_{\star} \sim \mathcal{O}(100), \quad g_{b} \sim \mathcal{O}(1)$ we have

$$
\frac{n_{B}}{s} \sim 0.01<\frac{T}{M}
$$

In the scenario of spontaneous baryogenesis, the baryon asymmetry is generated in thermal equilibrium. This requires baryon number violating interactions occur rapidly $\left(\Gamma_{\not B}>H\right)$. However if the B-violating interactions keep in equilibrium until $\dot{Q} \rightarrow 0$, the final baryon asymmetry will be zero. Denoting the epoch when the B-violating interactions freeze out by $T_{D}$, i.e. $\Gamma_{\not B}\left(T_{D}\right) \simeq H\left(T_{D}\right)$, the final baryon number asymmetry obtained is

$$
\left.\frac{n_{B}}{s}\right|_{T_{D}} \sim 0.01 c \frac{T_{D}}{M}
$$

For $T_{D}$ around the Grand Unification scale $10^{16} \mathrm{GeV}$, Eq.(13) shows that it is quite easy to have $n_{B} / s \sim 10^{-10}$ required by nucleosynthesis and measurement of Cosmic Microwave Background anisotropy, for both $M=M_{p l}$ and $M=M_{G U T}$. However, if the B-violating interactions of Grand Unified Theory conserve $B-L$, the asymmetry 
generated will be erased by the electroweak Sphaleron [16]. In this case Sphaleron $B+L$ interactions will make $T_{D}$ as low as around $100 \mathrm{GeV}$ and $n_{B} / s$ generated will be too small unless $c$ is unreasonably large.

Now we discuss the mechanism of leptogenesis [17 in our model by identifying $J^{\mu}$ with $J_{B-L}^{\mu}$ in Eq.(1). Doing the calculation with the same procedure as above for $J^{\mu}=J_{B}^{\mu}$ we have the final asymmetry of the baryon number minus lepton number

$$
\frac{n_{B-L}}{s} \sim 0.01 c \frac{T_{D}}{M} .
$$

The asymmetry $n_{B-L}$ in (14) will be converted to baryon number asymmetry when electroweak Sphaleron $B+L$ interaction is in thermal equilibrium which happens for temperature in the range of $10^{2} \mathrm{GeV} \sim 10^{12} \mathrm{GeV}$. $T_{D}$ in (14) is the temperature below which the $B-L$ violating interactions freeze out.

In the Standard Model of electroweak theory, $B-L$ symmetry is exactly conserved, however it could be violated by the (majorana) neutrino masses via higher dimensional effective operators. For instance, we take a dimension-5 operator,

$$
\mathcal{L}_{\psi}=\frac{2}{f} l_{L} l_{L} \phi \phi+h . c .,
$$

where $f$ is a scale of new physics beyond the Standard Model which generates the $B-L$ violations, $l_{L}$ and $\phi$ are the left-handed lepton and Higgs doublets respectively. When the Higgs field gets vacuum expectation value $\langle\phi\rangle \sim v$, the left-handed neutrino receives a majorana mass $m_{\nu} \sim \frac{v^{2}}{f}$.

In the early universe the lepton number violating rate induced by the interaction in (15) is [18]

$$
\Gamma_{\nvdash} \sim 0.04 \frac{T^{3}}{f^{2}}
$$

This rate at $T_{D}$ is required to be slower than the Universe expansion rate $\sim 1.66 g_{\star}^{1 / 2} T^{2} / M_{p l}$, which will put a lower limit on the scale $f$, consequently a upper limit on the neutrino mass $m_{\nu}$ [19].

To get the upper limit on $m_{\nu}$, we try to minimize the value of $f$. This can be done by minimizing the freezing out temperature $T_{D}$. From Eq.(14), one can see that if requiring $c \leq 4 \pi$ so that the effective lagrangian (Eq.(1)) is sensible for the perturbative calculation, $T_{D}$ must be larger than $10^{10} \mathrm{GeV}$ for $M=M_{p l}$, and $10^{7} \mathrm{GeV}$ for $M=$ $M_{G U T} \sim 10^{16} \mathrm{GeV}$. As a result of it, $f$ should be larger than about $10^{13} \mathrm{GeV}$ and the neutrino mass should be smaller than $\sim 4 \mathrm{eV}$ for $M=M_{p l}$. In the case that $M=M_{G U T}, f>4.9 \times 10^{11} \mathrm{GeV}$ and $m_{\nu}<0.1 \mathrm{keV}$. And for both cases, $T_{D}$ are much smaller than the cut-off scales. This makes our approximation of neglecting the r.h.s of Eq.(6) valid. We have also done a numerical calculation with result which supports for it.

So far what we have considered is the case that Quintessence is homogeneous in space. Since Quintessence is a scalar field one expects its fluctuations in space-time. In the following we study the isocurvature baryon number fluctuations generated in our model.

Since the baryon number asymmetry is proportional to $\dot{Q}$ and in the tracking regime $\dot{Q} \propto \sqrt{V(Q)}$, we have

$$
\frac{n_{B}}{s} \propto \sqrt{V(Q)}
$$

and

$$
\begin{gathered}
\delta\left(\frac{n_{B}}{s}\right) \propto \frac{V^{\prime}}{2 \sqrt{V}} \delta Q, \\
\frac{\delta\left(n_{B} / s\right)}{n_{B} / s}=\frac{V^{\prime}}{2 V} \delta Q .
\end{gathered}
$$

To get $\delta Q$, we are solving the equation of motion given below,

$$
\delta \ddot{Q}_{k}+3 H \delta \dot{Q}_{k}+\frac{k^{2}}{a^{2}} \delta Q_{k}+V^{\prime \prime} \delta Q_{k}=0,
$$

where $\delta Q_{k}$ is the Fourier transform of $\delta Q$. To have an analytical formula for $\delta Q$ we closely follow [20 22] by writing $V^{\prime \prime}$ as

$$
V^{\prime \prime}=\frac{3}{2} H \dot{c}_{Q}^{2}+\frac{9}{4}\left(1-c_{Q}^{2}\right)\left(w_{B}+c_{Q}^{2}+2\right) H^{2}
$$


where $c_{Q}^{2}$ is the sound speed of Quintessence defined by

$$
c_{Q}^{2} \equiv \frac{\dot{p}_{Q}}{\dot{\rho}_{Q}}=w_{Q}-\frac{\dot{w}_{Q}}{3 H\left(1+w_{Q}\right)} .
$$

Consider the case that the sound speed is approximately constant [21], the first term of the rhs. of Eq.(20) can be dropped out, then the solution to Eq.(19) is [21]:

$$
\delta Q_{k} \simeq \eta^{-1 / 2}\left[C_{1} J_{\nu}(k \eta)+C_{2} J_{-\nu}(k \eta)\right],
$$

where $\eta$ is the conformal time $\left(d \eta \equiv \frac{d t}{a}\right)$, and $a \propto \eta \propto T^{-1}$ during the radiation dominated era. In Eq.(22), $J_{ \pm \nu}(k \eta)$ are the first kind of Bessel functions, $C_{1}, C_{2}$ are constants which are time independent, $\nu^{2}$ and $\beta$ are,

$$
\begin{aligned}
\nu^{2} & \equiv \frac{1}{4}-\beta, \\
\beta & \equiv \frac{9}{4}\left(1-c_{Q}^{2}\right)\left(w_{B}+c_{Q}^{2}+2\right) .
\end{aligned}
$$

The modes of fluctuations which are interesting in cosmology are those outside the Hubble radius in very early universe, i.e. $k \eta \ll 1$. In such a limit the Bessel function can be approximated by

$$
J_{\nu}(k \eta) \simeq \frac{(k \eta)^{\nu}}{2^{\nu} \Gamma(1+\nu)},(k \eta \rightarrow 0) .
$$

On the initial value of $\delta Q_{k}$ denoted by $\delta Q_{k i}$, we assume the primordial fluctuation of the Quintessence scalar is generated during inflation. Being almost massless $\delta Q_{k i}$ is simply

$$
\left|\delta Q_{k i}\right|=\frac{H_{i n}}{\sqrt{2 k^{3}}}
$$

where $H_{\text {in }}$ is the Hubble constant during inflation. Furthermore the kinetic energy of the Quintessence will be diluted by inflation. Thus the Quintessence energy density remains potential energy dominated after inflation until the time $\eta_{t}$ when it goes into the tracking regime. This period of time is called the potential phase in the Ref. [21]. During this period $\rho_{Q}$ is nearly constant and $c_{Q}^{2} \simeq-w_{B}-2$ [2], hence $\beta \simeq 0$ and $V^{\prime \prime} \simeq 0$. We can see that $\delta Q_{k}$ is constant in the potential phase. But in the regime of tracking $\left(\eta \geq \eta_{t}\right)$,

$$
\begin{aligned}
c_{Q}^{2} & =w_{Q}, \\
\beta & =\frac{9}{4}\left(1-w_{Q}\right)\left(w_{B}+w_{Q}+2\right)=\frac{9}{4}\left(1-w_{Q}\right)\left(\frac{7}{3}+w_{Q}\right) .
\end{aligned}
$$

Numerically $-1<w_{Q} \leq 1 / 3$, so we have $\beta>1 / 4$. We obtain that

$$
\begin{aligned}
& \nu^{2}<0, \\
& \nu=i|\nu|=i \sqrt{\beta-\frac{1}{4}} \\
& J_{ \pm \nu}(k \eta) \simeq \frac{\exp \left( \pm i|\nu| \ln \frac{k \eta}{2}\right)}{\Gamma(1 \pm i|\nu|)} .
\end{aligned}
$$

Substituting $J_{ \pm \nu}(k \eta)$ above and $\delta Q_{k i}\left(\mathrm{Eq} \cdot(25)\right.$ ) into (22), we will get $\delta Q_{k}$.

We have also numerically solved Eq.(19) for the Quintessence model we use in this paper. In Fig.1 we plot $\delta Q_{k}$ as a function of red-shift $z$. One can see that $\delta Q_{k}$ is constant in the potential phase i.e. $\delta Q_{k i}=\delta Q_{k t}$, then oscillates with amplitude which decays like $\propto \eta^{-1 / 2}$ during tracking. This confirms our analytical analysis above.

Denoting the $\delta Q_{k}$ at the temperature $T_{D}$ when the baryon number asymmetry freezes in by $\delta Q_{k D}$, we have the ratio of $\left|\delta Q_{k D}\right|$ to the $\left|\delta Q_{k i}\right|$

$$
\left|\frac{\delta Q_{k D}}{\delta Q_{k i}}\right| \leq\left(\frac{\eta_{t}}{\eta_{D}}\right)^{1 / 2}=\left(\frac{T_{D}}{T_{t}}\right)^{1 / 2},
$$

where $T_{t}$ is the temperature when $Q$ transits from the potential phase to the tracking regime. Making use of the initial value of $\delta Q_{k}$ generated during inflation, $\delta Q_{k i}=\frac{H_{i n}}{\sqrt{2 k^{3}}}$, we have 


$$
\left|\delta Q_{k D}\right| \leq \frac{H_{i n}}{\sqrt{2 k^{3}}}\left(\frac{T_{D}}{T_{t}}\right)^{1 / 2} .
$$

Following [10], we calculate the RMS fluctuation of baryon number per logarithmic interval in our model which is given by

$$
\left(\frac{\delta n_{B}}{n_{B}}\right)_{k} \leq \frac{H_{i n}}{4 \pi}\left|V^{\prime}\left(Q_{D}\right) / V\left(Q_{D}\right)\right|\left(\frac{T_{D}}{T_{t}}\right)^{1 / 2}
$$

For the Quintessence model with potential in Eq.(8),

$$
\begin{aligned}
\left(\frac{\delta n_{B}}{n_{B}}\right)_{k} & \lesssim \frac{\lambda}{4 \pi} \frac{H_{i n}}{M_{p l}}\left(\frac{T_{D}}{T_{t}}\right)^{1 / 2} \\
& \leq 10^{-5}\left(\frac{T_{D}}{T_{t}}\right)^{1 / 2}
\end{aligned}
$$

which will be smaller than $10^{-5}$ since $T_{t}>T_{D}$. To get the result above we have used the constraints on $\frac{H_{i n}}{M_{p l}} \leq 10^{-5}$ 23] from the limit of the detecting of primordial gravitational waves predicted by inflation models. In addition we have also taken $\lambda^{2} \sim \mathcal{O}(10)$ to satisfy the constraints from the nucleosynthesis. The isocurvature baryon number fluctuation in (31) is small and is not conflict with the Boomerang and Maxima-1 data on the anisotropy of cosmic microwave background 24].

The isocurvature baryon number fluctuation in the spontaneous baryogenesis [9] has been studied by Turner, Cohen and Kaplan [10], where they find the amplitude sizable. In their model the potential of the ilion field $\theta$ is $m^{2} \theta^{2}$. The produced baryon asymmetry is linearly proportional to the initial value of the ilion field $\theta_{0}$. Therefore quantum fluctuations in the initial $\theta_{0}$ during inflation will directly induce isocurvature baryon number fluctuations. In our model the Quintessence has the tracking behavior, and the produced baryon number asymmetry is almost independent of the initial conditions of Quintessence field. Consequently a small isocurvature perturbation is expectable.

The tracking behavior of the Quintessence, however plays an important role in our discussions. Because of the tracking, $\dot{Q}$ can be large enough and the baryon number asymmetry required can be generated. For models without tracking property, such as the model with potential $V(Q)=\Lambda^{4}\left(1 \pm \cos \frac{Q}{M}\right)$, we have checked and found that $\dot{Q}$ is too small to account for $n_{B} / s \sim 10^{-10}$.

Our mechanism for leptogenesis, however does put a lower limit on the reheating temperature $T_{R}$. It requires $T_{R}$ be higher than $T_{t}$ and $T_{D}$. For cut-off $M$ of Eq.(1) in the range of $10^{16} \sim 10^{19} \mathrm{GeV}, T_{D}$ is about $10^{7} \sim 10^{10} \mathrm{GeV}$, which will not put a strong constraint on models of inflation and reheating.

In summary we have introduced in this paper an effective interaction of the Quintessence scalar to the ordinary matter and studied the possibilities of baryogenesis. Our model can naturally explain the baryon number asymmetry of our Universe $n_{B} / s \sim 10^{-10}$ via spontaneous leptogenesis. Our scenario provides a unified description for the present accelerated expansion and baryon number asymmetry generation of our Universe by the dynamics of Quintessence.

Acknowledgments: We thank David Kaplan for correspondence on spontaneous baryogenesis and Robert Brandenberger, Fabio Finelli, Z-H. Lin for discussions. This work is supported in part by National Natural Science Foundation of China under Grant No. 10047004 and by Ministry of Science and Technology of China under Grant No. NKBRSF G19990754.

[1] S. Pelmutter et al., Astrophys. J. 483, 565 (1997).

[2] B. Ratra and P.J.E. Peebles, Phys. Rev. D37, 3406 (1988).

[3] C. Wetterich, Nucl. Phys. B302, 302 (1988).

[4] I. Zlatev, L. Wang and P.J. Steinhardt, Phys. Rev. Lett, 82, 896 (1999); P.J. Steinhardt, L. Wang and I. Zlatev, Phys. Rev. D59, 123504 (1999).

[5] M.S. Turner, astro-ph/0108103.

[6] S.M. Carroll, astro-ph/0107571; R.R. Caldwell, Braz. J. Phys. 30, 215 (2000).

[7] R.D. Peccei, hep-ph/0009030; C. Kolda, D.H. Lyth, Phys. Lett. B458, 197 (1999).

[8] S.M. Carroll, Phys. Rev. Lett, 81, 3067 (1998).

[9] A. Cohen and D. Kaplan, Phys. Lett. B199, 251 (1987).

[10] M.S. Turner, A. Cohen and D. Kaplan, Phys. Lett. B216, 20 (1989). 
[11] A. Albrecht and C. Skordis, Phys. Rev. Lett, 84, 2076 (2000); C. Skordis and A. Albrecht, astro-ph/0012195.

[12] J.J. Halliwell, Phys. Lett. B185, 341 (1987); J. Barrow, Phys. Lett. B187, 12 (1987).

[13] A.R. Liddle and R.J. Scherrer, Phys. Rev. D59, 023509 (1998); E.J. Copeland, A.R. Liddle and D. Wands, Phys. Rev. D57, 4686 (1998).

[14] P. Ferreira and M. Joyce, Phys. Rev. D58, 023503 (1998).

[15] P. Binétruy, Phys. Rev. D60, 063502 (1999); P. Binétruy, Int. J. Theor. Phys. 39, 1859 (2000).

[16] N. Manton, Phys. Rev. D28, 2019 (1983); F. Klinkhamer and N. Manton, Phys. Rev. D30, 2212 (1984); V.A. Kuzmin, V.A. Rubakov, and M.E. Shaposhnikov, Phys. Lett. B155, 36 (1985).

[17] M. Fukugida and T. Yanagida, Phys. Lett. B174, 45 (1986); P. Langacker, R.D. Peccei, and T. Yanagida, Mod. Phys. Lett. A1, 541 (1986); M. Luty, Phys. Rev. D45, 455 (1992); R.N. Mohapatra and X. Zhang, Phys. Rev. D46, 5331 (1992).

[18] U. Sarkar, hep-ph/9809209; W. Buchmuller, hep-ph/0101102.

[19] M. Fukugida and T. Yanagida, Phys. Rev. D42, 1285 (1990); S.M. Barr and A. Nelson, Phys. Lett. B246, 141 (1991).

[20] P. Brax, J. Martin and A. Riazuelo, Phys. Rev. D62, 103505 (2000).

[21] L.R. Abramo and F. Finelli, Phys. Rev. D64, 083513 (2001).

[22] M. Kawasaki, T. Moroi and T. Takahashi, astro-ph/0108081.

[23] D. Lyth and A. Riotto, Phys. Rept. 314, 1 (1999); X. Wang, M. Tegmark and M. Zaldarriaga, astro-ph/0105091.

[24] K. Enqvist, H. Kurki-Suonio and J. Väliviita, Phys. Rev. D62, 103003 (2000); M. Bucher, K. Moodley and N. Turok, astro-ph/0012141; R. Trotta, A. Riazuelo and R. Durrer, Phys. Rev. Lett. 87, 231301 (2001). 


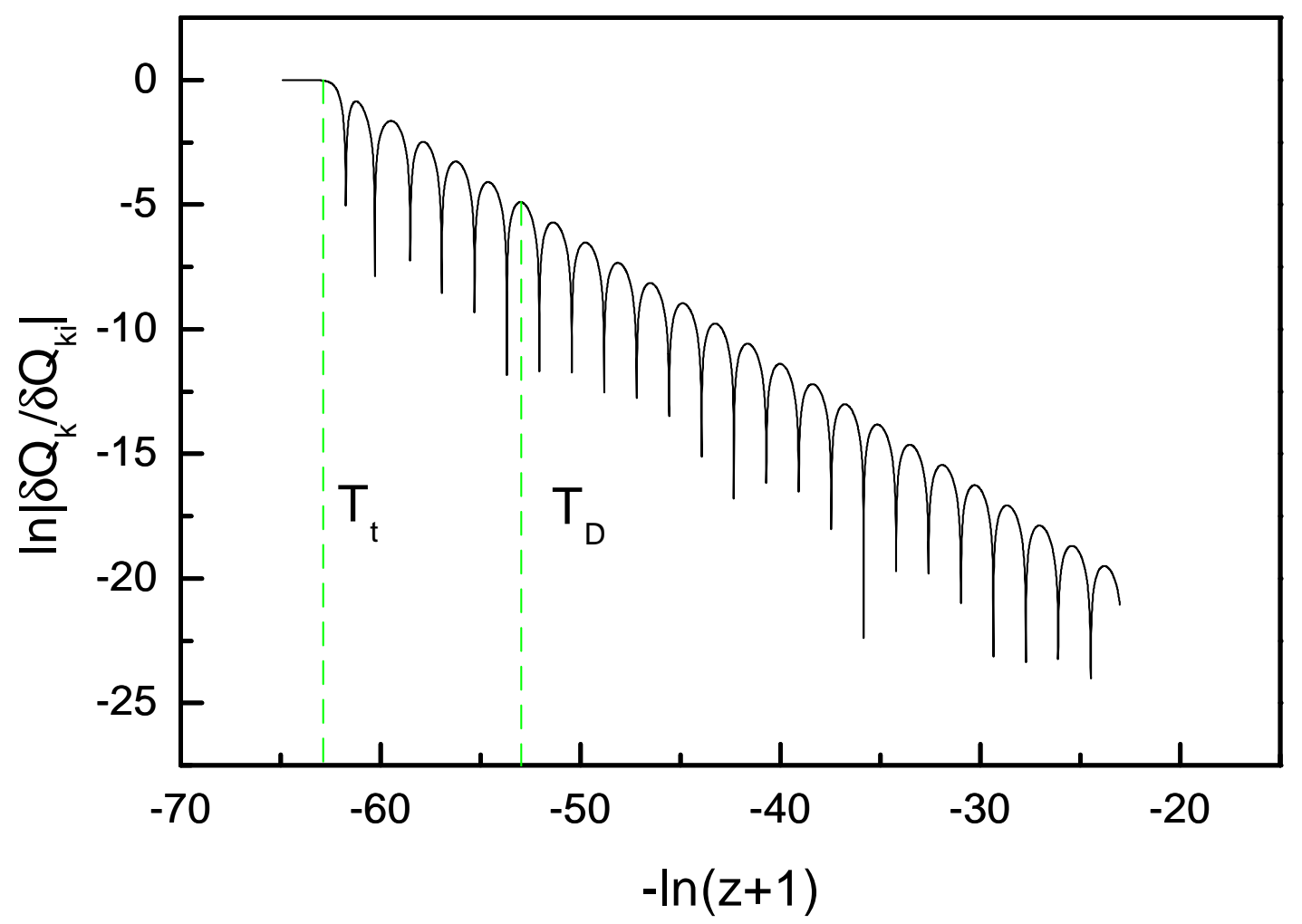

FIG. 1. Plot of $\delta Q_{k}$ as function of red-shift $z$. The $x$-axis is $-\ln (z+1)$, and the $y$-axis is $\ln \left|\delta Q_{k} / \delta Q_{k i}\right|$. In this plot, $T_{t}$ corresponds to temperature around $10^{14} \mathrm{GeV}$ and $T_{D} \simeq 10^{10} \mathrm{GeV}$. 